\title{
Impaired Immune Phenotype of Endothelial Cell-derived Micro Particles: The Missing Link between Diabetes-related States and Risk of Cardiovascular Complications?
}

\section{Alexander E Berezin}

Internal Medicine Department, State Medical University of Zaporozhye, Mayakovsky, Zaporozhye, UA-69035, Ukraine

Corresponding author: Alexander E Berezin, Internal Medicine Department, State Medical University of Zaporozhye, Mayakovsky, Zaporozhye, UA-69035, Ukraine, Tel: +380612894585; E-mail: dr_berezin@mail.ru; aeberezin@gmail.com

Received date: April 11, 2016; Accepted date: April 21, 2016; Published date: April 28, 2016

Copyright: (C) 2016 Berezin AE. This is an open-access article distributed under the terms of the Creative Commons Attribution License, which permits unrestricted use, distribution, and reproduction in any medium, provided the original author and source are credited.

\begin{abstract}
Type two diabetes mellitus (T2DM) remains a leading contributor to cardiovascular (CV) mortality worldwide. Although obese and metabolic syndrome are discussed key factors contributing a higher risk of T2DM, the exact molecular mechanisms underlying to the progression of dysmetabolic states are still not completely clear. There is large body of evidence regarding endothelial dysfunction as a key player in increasing CV risk and that vascular damage in the dysmetabolic patients might mediate through an imbalance between various populations of micro particle (MP). The short commentary is discussed a role of impaired ratio between apoptotic MPs and activated MPs derived from endothelial cells that was recognized as "impaired phenotype" of endothelial cell-derived MPs. It has considered the causality epigenetic reprogramming, metabolic disorders, inflammation, and oxidative stress in forming of "impaired phenotype" of MPs. The incorporation of measurement of the endothelial cell-derived MP number into a conventional CV risk factor model aimed improving of risk stratification of the dysmetabolic patients with high probability of CV disease is discussed.
\end{abstract}

Keywords: Diabetes mellitus; Obesity; Metabolic syndrome; Circulating endothelial cell derived microparticles; Cardiovascular risk factors

\section{Review}

Type two diabetes mellitus (T2DM) is discussed a major global epidemic leading to loss health in general population [1]. Although the results of recent studies have been well established that overweigh/ obesity and metabolic syndrome are represented the leading risk factor for T2DM and diabetes-related cardiovascular (CV) complications [2-4], the impact of the dysmetabolic states on molecular mechanisms regarding vasculature repair systems and "metabolic memory" is not fully investigated [5-7]. By now, endothelial dysfunction (ED) has found a key player in increasing CV risk in overweigh/obese and T2DM subjects $[8,9]$. Furthermore, ED interacting with worse glucose metabolism, lipotoxicity, over production of free radicals and other components of oxidative stress contributes to increase CV risk $[9,10]$ Based on current available evidence cell-to-cell cooperation is crucial for various biological responses including thrombogenicity, coagulation, inflammation, immunity, cell growth and differentiation, vascular and plaque remodeling [11]. In this context, extracellular microparticles (MPs) acting as detrimental regulator of cell-to-cell cooperation are discussed a have as a key marker of global vascular damage in dysmetabolic patients [12]

MPs (microvesicles with size $<1000 \mathrm{~nm}$ ) are released from plasma membrane of wide variety of cells including red blood cells, mononuclears, platelets, endothelial cells, resulting in specific (cytokine and growth stimulation, apoptotic agents, coagulation, active molecules' effect) and non-specific (shear stress) stimuli [13]. MPs coordinate wide spectrum biological processes, i.e., angiogenesis, neovascularization, cell growth/differentiation, proliferation, coagulation, and they are involved in the epigenetic regulation of post- processing that is essential for phenotype modification, tissue repair, cell death, malignancy, and immunity [14-17].

Numbers of circulating endothelial cell-derived microparticles (EMPs) were found a marker of endothelial dysfunction and predictor of CV complications in dysmetabolic subjects including obesity and T2DM [18-22]. Moreover, several patterns of circulating microvesicles were found a predictor of clinical outcomes in patients with known CV disease [20,22]. All these findings illustrate a wide spectrum of biological functions of EMPs affecting pathogenesis of metabolic and CV diseases.

Interestingly, EMPs depending on their origin (apoptotic cells or shedding from activated endothelial cell) might have a different structure and produce controversial effects. Apoptotic-derived EMPs are capable of transferring biological information (regulating peptides, active molecules, hormones) or even genetic materials (micro-RNA, mRNA, and DNA), as well as proteins, lipid components, from one cell to another without direct cell-to-cell contact to maintain cell homeostasis [23]. Apoptotic EMPs may be not only immune mediators, generating powerful signaling by the simultaneous receptor interaction, but they are discussed a marker of endothelial cell injury, coagulation/thrombosis and vascular aging [24]. Contrary, EMPs derived from activated endothelial cells did not contain nuclear components and they may exhibit angiogenic properties and contribute to tissue protection $[25,26]$. It has been suggested that an ability of various cells to secrete micropartilces may depend on epigenetic modifications and that this cell phenotype alteration by cellderived vesicles presents a new aspect for consideration of dysmetabolic state development [27]. 
Page 2 of 3

Recently it has reported that patients with obesity, metabolic syndrome and T2DM may have impaired ratio between number of apoptotic EMPs and EMPs derived from activated endothelial cells $[19,20]$. This imbalance was predominantly associated with increased number of apoptotic EMPs labeled as CD31+/annexin V+, whereas number of activated EMPs phenotypes as CD62E+ did not change or appeared to be tendency to decrease [28]. Indeed, elevated CD31+/ annexin $\mathrm{V}+$ to $\mathrm{CD} 62 \mathrm{E}+$ ratio was fond as indicator of impaired immune phenotype of EMPs, which allows determining pattern of microparticles in dysmetabolic disorder patients [29]. This phenomenon was recognized as "impaired phenotype" of EMPs and it has related to cellular injury, inflammation, coagulation/thrombosis leading to vascular dysfunction and contributing to CV risk [28-31]. Finally, "impaired phenotype" of EMPs is appearing as epigenetic reprogramming of matter' cells play a pivotal role in the development of CV complications in T2DM [32-34].

Although there was a skepticism regarding origin of imbalance in several poles of EMP in patients with obesity and diabetes, it has supposed that inflammatory cytokine and probably lipid abnormalities may consider a possible cause of predominantly immune phenotype of EMPs $[35,36]$. The skepticism is based on findings regarding a pivotal role of increased glucose level, inflammatory cytokine and lipid abnormalities in the development of impaired phenotypes seen in T2DM patients. Indeed, glucose toxicity and lipid toxicity are recognized as main contributors of metabolic memory affected epigenetic reprogramming of various types of progenitor cells. By now, endothelial progenitor cells have been found as component of endogenous repair system, which mediates tissue repair including microvasculature and microvasculature damages [27]. There are current available data regarding that the EMPs interact with or enter different target cells from other tissues, altering their phenotype toward that of the cell releasing the vesicles [27]. Cells may be changed by direct interactions with microvesicles, transfer of cell surface receptors or directly epigenetic reprogramming via transcriptional regulators derived with circulating microparticles. Thus, EMPs are essential for cross-communication between cells and they may underlie the phenomenon of tissue reparation.

Additionally, the circulating EMPs are involved in the cell-to-cell cooperation supporting mobbing and differentiation of the progenitor cells. Moreover, the dysfunction of progenitor cells has been recently described as an essential factor contributed to microvascular and microvascular complications in diabetes, metabolic syndrome and obesity. In this context, "impaired phenotype" of EMPs is a link between $\mathrm{CV}$ risk factors and epigenetic reprogrammed progenitor cells.

Obviously patients with various dysmetabolic disorders might have different EMP' patterns contributing to the development of CV complications depending on the type of reprogramming of matter' cells. Whether abnormal pattern of EMPs would be appeared prior to metabolic states manifestation or, in contrast, several metabolic states (i.e., diabetes, metabolic syndrome, obesity) are able to induce an imbalance between various EPMs via epigenetic reprogramming mechanisms affected matter' cells secreting microvesicles is not still clear and requires more investigations. Probably, incorporation of the EMP level into a conventional risk factor model is able to be useful tool for improving of risk stratification of the dysmetabolic patients with high probability of CV disease [37-39].

Importantly, the accurate assessment of MPs remains a serious challenge because of a lack of consensus regarding methodologies to measure MPs and the inability of most techniques to capture the entire size range of these vesicles. The conventional approach for measuring the MPs is based on commonly used flow cytometry and MP tracking analysis that recognized as a gold standard, as well as Western blot analysis and electron microscopy [40]. However, a lot of conventional methods have crucial limitations regarding complicated assay and suffers from relatively low sensitivity and accuracy because of resolution problems occurring for the majority of commercially available flow cytometers. The serious barrier created surmountable problems for MP tracking analysis is sizing of small MPs $(<50-100$ $\mathrm{nm}$ ). Western blot analysis and electron microscopy allow to optionally recognizing MPs depending on determination of different markers, represents a useful tool for examining particles. However, Western blot analysis and electron microscopy require subsequent technical efforts and are much expensive. Alternatively, the surface plasmon resonancebased imaging (SPRi) microscopy was recently recognized as a novel method for quantification and sizing of MPs, which could be significantly useful to resolve the majority problems affected MP' recognition. The high contrast in SPR signal between cell edges and substratum facilitates identification of cell edges and segmentation of cell areas. Therefore, Raman micro-spectroscopy, micro nuclear magnetic resonance technique, small-angle $\mathrm{X}$-ray scattering, and anomalous small-angle X-ray scattering might compete with SPRi microscopy and flow cytometery to increase sensitivity and accuracy in detecting of MPs [40].

\section{Conclusion}

In conclusion, circulating MPs have promise in the clinical arenas of dysmetabolic disease biomarkers. There are evidence in supporting of hypothesis that apoptotic-derived EMPs might be one of causality factor contributed to pathogenesis of obesity, metabolic syndrome and diabetes. The large clinical trials are required to explain the predictive role of circulating EMP subsets in dysmetabolic individuals at higher risk of CV complications.

\section{References}

1. Nichols GA, Schroeder EB, Karter AJ, Gregg EW, Desai J, et al. (2015) SUPREME-DM Study Group. Trends in Diabetes Incidence Among 7 Million Insured Adults, 2006-2011: The SUPREME-DM Project. Am J Epidemiol 181: 32-39.

2. King RJ, Grant PJ (2016) Diabetes and cardiovascular disease: pathophysiology of a life-threatening epidemic. Herz.

3. Kasim NB, Huri HZ, Vethakkan SR, Ibrahim L, Abdullah BM (2016) Genetic polymorphisms associated with overweight and obesity in uncontrolled Type 2 diabetes mellitus. Biomark Med 10: 403-415.

4. Izquierdo-Lahuerta A, Martínez-García C, Medina-Gómez G (2016) Lipotoxicity as a trigger factor of renal disease. J Nephrol.

5. Zhang Z, Zhang X, Dong J, Gao W, Liu F, et al. (2016) Association of chemokine ligand 5/chemokine receptor 5 gene promoter polymorphisms with diabetic microvascular complications: A meta-analysis. J Diabetes Investig 7: 212-218.

6. Tibaut M, Petrovič D (2016) Oxidative stress genes, antioxidants and coronary artery disease in type 2 diabetes mellitus. Cardiovasc Hematol Agents Med Chem.

7. Berezin A (2016) Metabolic memory phenomenon in diabetes mellitus: Achieving and perspectives. Diabetes Metab Syndr.

8. van Sloten TT, Henry RM, Dekker JM, Nijpels G, Unger T, et al. (2014) Endothelial dysfunction plays a key role in increasing cardiovascular risk in type 2 diabetes: the Hoorn study. Hypertension 64: 1299-1305. 
Citation: Berezin AE (2016) Impaired Immune Phenotype of Endothelial Cell-derived Micro Particles: The Missing Link between Diabetes-related States and Risk of Cardiovascular Complications?. J Data Mining Genomics \& Proteomics 7: 195. doi:10.4172/2153-0602.1000195

Page 3 of 3

9. Jia G, Sowers JR (2014) Endothelial dysfunction potentially interacts with impaired glucose metabolism to increase cardiovascular risk. Hypertension 64: 1192-1193.

10. Zaghloul A, Al-Bukhari TA, Al-Pakistani HA, Shalaby M, Halawani SH, et al. (2014) Soluble endothelial protein C receptor and high sensitivity C reactive protein levels as markers of endothelial dysfunction in patients with type 1 and type 2 diabetes mellitus: Their role in the prediction of vascular complications. Diabetes Res Clin Pract 106: 597-604.

11. Wu ZH, Ji CL, Li H, Qiu GX, Gao CJ, et al. (2013) Membrane microparticles and diseases. Eur Rev Med Pharmacol Sci 17: 2420-2427.

12. Barteneva NS, Fasler-Kan E, Bernimoulin M, Stern JN, Ponomarev ED, et al. (2013) IA. Circulating microparticles: square the circle. BMC Cell Biol 14: 23 .

13. Kurtzman N, Zhang L, French B, Jonas R, Bantly A, et al. (2013) Personalized cytomic assessment of vascular health: Evaluation of the vascular health profile in diabetes mellitus. Cytometry B Clin Cytom 84: 255-266.

14. Markiewicz M, Richard E, Marks N, Ludwicka-Bradley A (2013) Impact of endothelial microparticles on coagulation, inflammation, and angiogenesis in age-related vascular diseases. J Aging Res 2013: 734509.

15. Martinez MC, Andriantsitohaina R (2011) Microparticles in angiogenesis: therapeutic potential. Circ Res 109: 110-119.

16. Tetta C, Bruno S, Fonsato V, Deregibus MC, Camussi G (2011) The role of microvesicles in tissue repair. Organogenesis 7: 105-115.

17. Rautou PE, Vion AC, Amabile N, Chironi G, Simon A, et al. (2011) Microparticles, vascular function, and atherothrombosis. Circ Res 109: 593-606.

18. Berezin AE, Kremzer AA, Berezina TA, Martovitskaya YV (2015) Pattern of circulating microparticles in chronic heart failure patients with metabolic syndrome: Relevance to neurohumoral and inflammatory activation. BBA Clin 4: 69-75.

19. Berezin AE, Kremzer AA, Samura TA, Berezina TA, Kruzliak P (2015) Impaired immune phenotype of circulating endothelial-derived microparticles in patients with metabolic syndrome and diabetes mellitus. J Endocrinol Invest 38: 865-874.

20. Berezin A, Kremzer A, Berezina T, Martovitskaya Y, Gromenko O (2015) The pattern of circulating microparticles in diabetes mellitus patients with known subclinical atherosclerosis. Clin Med Biochemistry 1: 104-110.

21. Leroyer AS, Tedgui A, Boulanger CM (2008) Microparticles and type 2 diabetes. Diabetes Metab 34: S27-S32.

22. Amabile N, Cheng S, Renard JM, Larson MG, Ghorbani A, et al. (2014) Association of circulating endothelial microparticles with cardiometabolic risk factors in the Framingham Heart Study. Eur Heart J 35: 2972-2979.

23. Nomura S (2009) Dynamic role of microparticles in type 2 diabetes mellitus. Curr Diabetes Rev 5: 245-251.

24. Puddu P, Puddu GM, Cravero E, Muscari S, Muscari A (2010) The involvement of circulating microparticles in inflammation, coagulation and cardiovascular diseases. Can J Cardiol 26: 140-145.
25. Jimenez JJ, Jy W, Mauro LM, Soderland C, Horstman LL, et al. (2003) Endothelial cells release phenotypically and quantitatively distinct microparticles in activation and apoptosis. Thrombosis Research 109: $175-180$

26. Arteaga RB, Chirinos JA, Soriano AO, Jy W, Horstman L, et al. (2006) Endothelial microparticles and platelet and leukocyte activation in patients with the metabolic syndrome. Am J Cardiology 98: 70-74.

27. Quesenberry PJ, Aliotta JM (2010) Cellular phenotype switching and microvesicles. Adv Drug Deliv Rev 62: 1141-1148.

28. Berezin AE (2015) Are Endothelial Cell-Derived Microparticles Predictive Biomarkers in Cardiovascular Diseases? Atherosclerosis: Open Access 1: e101-e103.

29. Berezin AE, Kremzer AA, Samura TA, Berezina TA, Kruzliak P (2015) Immune phenotypes of endothelial-derived microparticles in dysmetabolic patients. Journal of Proteomics \& Bioinformatics 8: 060-066.

30. Chironi GN, Boulanger CM, Simon A, Dignat-George F, Freyssinet JM, et al. (2009) Endothelial microparticles in diseases. Cell and Tissue Research 335: 143-151.

31. Shantsila E (2009) Endothelial microparticles: a universal marker of vascular health? J Hum Hypertens 23: 359-361.

32. Camussi G, Deregibus MC, Bruno S, Grange C, Fonsato V, et al. (2011) Exosome/microvesicle mediated epigenetic reprogramming of cells. Am J Cancer Research 1: 98-110.

33. Théry C, Ostrowski M, Segura E (2009) Membrane vesicles as conveyors of immune responses. Nat Rev Immunol 9: 581-593.

34. Wang Y, Chen LM, Liu ML (2014) Microvesicles and diabetic complications - novel mediators, potential biomarkers and therapeutic targets. Acta Pharmacol Sin 35: 433-443.

35. Mause SF, Weber C (2010) Microparticles: protagonists of a novel communication network for intercellular information exchange. Circ Res 107: 1047-1057.

36. Montoro-García S, Shantsila E, Marín F, Blann A, Lip GY (2011) Circulating microparticles: new insights into the biochemical basis of microparticle release and activity. Basic Res Cardiol 106: 911-923.

37. Berezin AE (2015) Circulating Endothelial-Derived Apoptotic Microparticles as Novel Perspective Biomarker for Diabetes. Diabetes Res Treat Open Access 1: 117-120.

38. Berezin AE, Kremzer AA, Martovitskaya YV, Berezina TA, Gromenko EA (2016) Pattern of endothelial progenitor cells and apoptotic endothelial cell-derived microparticles in chronic heart failure patients with preserved and reduced left ventricular ejection fraction. EBioMedicine 4: 86-94.

39. Berezin AE, Kremzer AA, Martovitskaya YV, Samura TA, Berezina TA (2014) The Biomarker Risk Prediction Score in Chronic Heart Failure. J Dis Markers 1: 8-15.

40. Berezin AE (2015) The development of biological molecular sensing techniques to detect microparticles: focus on clinical medicine benefits. J Microb Biochem Technol 7: 236-237. 